\title{
Research on the Power Prediction and Harmonic Evaluation of Electric Vehicle in Residential Area
}

\author{
Linghan Zhang', a, Yuanyuan Sun ${ }^{1, \mathrm{~b}}$, Tao $\mathrm{Yu}^{2, \mathrm{c}}$, Shoubin Diao ${ }^{3, \mathrm{~d}}$, Yonggang Zhu ${ }^{3, \mathrm{e}}$ \\ ${ }^{1}$ School of electrical engineering, Shandong University, Jinan 250061, China. \\ ${ }^{2}$ Jinan Urban Planning and Design Institute, Jinan 250001, China. \\ ${ }^{3}$ Lichen Power Supply Company of National Grid, Jinan 250100, China. \\ a530278792@qq.com, ${ }^{b}$ sunyy@sdu.edu.cn, ${ }^{c}$ ghyyt@163.com, ${ }^{d}$ diaoshoubin@163.com, ${ }^{e}$ zhu_yg@ho \\ tmail.com
}

Keywords: Power Prediction, Electric Vehicle, Harmonic Evaluation

\begin{abstract}
The development of electric vehicles (EVs) as a new energy vehicle is increasing. The charging power and harmonic prediction are the basis for analyzing EV operation scheduling and improving power quality. Firstly, the Monte Carlo method is uesd to obtain the initial charging time, daily driving mileage, and the stage of charge (SOC) of the electric vehicle. The charging duration of the EV can be obtained according to different charging power and battery performance. Then based on the charging power varying with SOC, the harmonic coupling admittance matrix staged model can be established. Based on the power and harmonic current models, the charging power and harmonic current of single and large scale EVs in residential areas are determined. Finally, the results indicate that the charging load increases the peak-to-valley difference and rises the total harmonic distortion (THD) of the residential area.
\end{abstract}

\section{Introduction}

In the context of energy shortages and serious environmental pollution, the market share of EVs has increased year by year [1]. EVs have high charging power and high harmonic current, and when the scale of development increases, the grid will be greatly affected. EV charging power and harmonic prediction can provide decision-making basis for power grid dispatching and improving power quality.

In [2], under a single charging power, it is assumed that the EV is randomly charged during a fixed period of time to predict the charging situation of the EV. Reference [3] assumes that the charging time of EVs is fixed, and the charging load is predicted based on the constant power model. Reference [4] studied the charging load time of EVs with a fixed capacity and a single type of battery. However, the charging method and power are varied during the actual charging process of EVs, and the performance of different electric vehicle batteries is different. The charging process is divided into two stages of constant current-constant voltage, not constant power charging.

In view of the above deficiencies, this paper divides the different charging behavior and charging power of EVs with different battery performances. An electric vehicle charging time calculation model based on Monte Carlo method is proposed. The stage changing power and harmonic 
coupling admittance matrix current model is established, considering the constant current-constant voltage charging process of EVs. The charging situation of EV in residential area and its influence on residential electricity consumption are also analyzed.

\section{The Electric Vehicle Travel Rules and Harmonic Models}

\subsection{Electric Vehicle Charging Load Characteristics}

The way electric vehicles are charged from the grid is usually divided into two situations: regular charging and fast charging. Regular charging time is longer and generally lasts 5-8 hours. In this charging mode, users can choose charging time and space can be selected by users more freely and flexibly, and the general power users' demand can be satisfied. The fast charging current is 10 times or more of regular charging, and the typical charging time is 1-2 hours, but excessive charging current will adversely affect the power grid.

The different brands of electric vehicles have different battery types, and the corresponding battery capacity and maximum cruising range are different. The charging time of the electric vehicle is affected, and the actual charging curve is also different, but the overall charging curve has the same trend. Lithium batteries are often charged by a two-stage charging method combining constant current and constant voltage. The charging current is controlled to keep it at a fixed value during the constant current charging phase. When the battery reaches the maximum allowable charging voltage, the constant voltage phase will be entered. At this stage, the charging voltage of the battery is kept constant, and the charging current of the battery is reduced to zero in a hyperbolic trend.

\subsection{Analysis of Charging Behavior of EVs}

The travel rules of EVs mainly include: initial charging time, battery residual state and EV daily mileage, etc., which are closely related to the activity chain of electric vehicle users in one day.

\subsubsection{Charging start time}

The travel time of the owner's weekday is mainly commuting activities, while the weekend is entertainment activities, which leads to a large difference in charging behavior between weekends and weekdays. According to the statistics of the 2009 National Household Travel Survey database [5], the fitting results of the charging time distribution of the private car users are determined separately in the residential area on weekdays and weekends, and basically obey the normal distribution as shown in equation (1).

$$
f(t)= \begin{cases}\exp \left(-\left(x-\mu_{t}\right)^{2} /\left(2 \sigma^{2}\right)\right) /(\sigma \sqrt{2 \pi}) & \mu_{t}-12<x \leq 24 \\ \exp \left(-\left(x+24-\mu_{t}\right)^{2} /\left(2 \sigma^{2}\right)\right) /(\sigma \sqrt{2 \pi}) & 0<x \leq \mu_{t}-12\end{cases}
$$

Where $f(t)$ is the probability density of the charging start time; $\mu_{t}$ and $\sigma$ are the mean and standard deviation of the charging start time.

\subsubsection{Daily mileage}

The method for estimating the maximum likelihood of the daily mileage is used [6], and the statistical data is normalized by analyzing the daily mileage of the electric vehicle. The daily travel mileage is approximated as a lognormal distribution, and the probability distribution function is as shown in equation (2). The parameter values are obtained according to the NHTS data survey, and 
are adjusted accordingly by combining the specific conditions of electric vehicles in different cities or regions and the development scale of future social electric vehicles.

$$
\begin{gathered}
d(x)=\exp \left(-\left(\ln x-\mu_{x}\right)^{2} /\left(2 \sigma_{x}^{2}\right)\right) /\left(\sqrt{2 \pi} x \sigma_{x}\right) \\
\text { s.t. } \quad \mu_{x}=\ln \left(E_{x}\right)-\ln \left(1+D_{x} / E_{x}^{2}\right) / 2 \\
\sigma_{x}^{2}=\ln \left(1+D_{x} / E_{x}^{2}\right)
\end{gathered}
$$

Where $\mu_{x}$ is the average value; $\sigma_{x}$ is the standard value of the logarithm of the variable; $E_{x}$ and $D_{x}$ are the expected and variance.

\subsubsection{Stage of charge}

The remaining battery power of the electric vehicle is used to measure the charging demand of the EV. Assuming that the battery consumption is proportional to the distance traveled, the remaining power at the end of the last trip can be calculated by the following equation (3).

$$
\begin{gathered}
S O C=\left\{\begin{array}{cc}
S_{S O C_{O}-m / r_{m}} & 0 \leq m \leq r \\
0 & m>r
\end{array}\right. \\
\text { s.t. } \quad r=r_{m} \times S O C_{o}
\end{gathered}
$$

Where SOC $_{O}$ is the remaining capacity of the EV after the last charge; $m$ is the mileage of the $\mathrm{EV} ; r_{m}$ and $\mathrm{r}$ are the maximum cruising range at full power and mileage of electric vehicles.

\subsubsection{Charging duration}

According to the charging power corresponding to different SOC, the charging process of the electric vehicle is divided into $\Delta t$ stages. The charging duration of each stage is calculated by the formula (4)-(5).

$$
\begin{aligned}
t_{i} & =\left[C \times\left(S O C_{i}-S O C_{i-1}\right)\right] /\left(P_{i} \times \eta\right) \\
t_{c}= & \sum_{i=1}^{\Delta t} t_{i}
\end{aligned}
$$

Where $\mathrm{SOC}_{\mathrm{i}}$ is the remaining battery power of stage $i$; $C$ is the battery capacity; $P_{i}$ is the charging power for each stage; $\eta$ is the efficiency of the charger, usually taken at $90 \%$.

\subsection{Electric Vehicle Electrical Model}

Single-phase or three-phase rectifier circuits are often used in electric vehicle chargers. Passive power factor corrected single-phase and three-phase uncontrolled rectifier chargers are used in this paper. The equivalent circuit filter inductor $L$ and filter capacitor $C$ parameter estimates are obtained, through charging current characteristics analysis and charger feature data extraction. The resistance value $R$ is obtained according to the charging power under different charging conditions. The harmonic equivalent circuit of the charger is analyzed, and the harmonic coupling admittance matrix model of the electric vehicle charger is obtained.

\section{Electric Vehicle Charging Load Power and Harmonic Evaluation}

The Monte Carlo method is used to simulate the charging demand of electric vehicles, by analyzing the behavior of the charging load and the probability distribution characteristics. The flow is shown in Figure 1. 


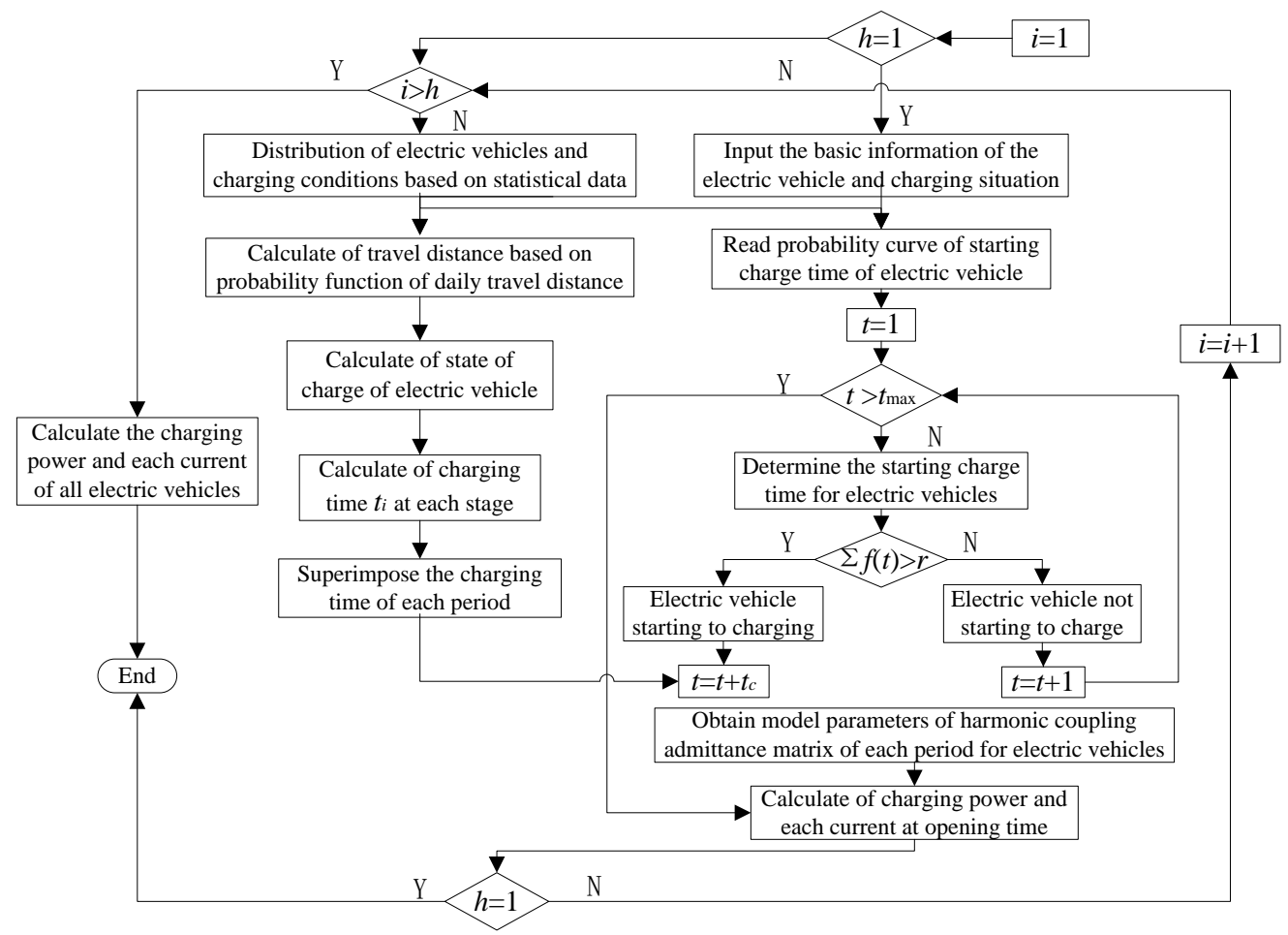

Figure 1. EV power and harmonic prediction flow chart.

Step 1) Enter the basic information of the electric vehicle, including: electric vehicle battery capacity, maximum cruising range, charging method and charging power.

Step 2) The access time of the electric vehicle is determined, and the charging start time probability curve corresponding to the electric vehicle is selected according to the weekend or not. The initial charging time of the electric vehicle is calculated by the formula (1) through the method of Monte Carlo.

Step 3) The initial power SOC $_{O}$ is selected before the electric vehicle travels. According to the probability density $d(m)$ of the driving distance, the travel distance is determined, and the initial battery state of charge of the electric vehicle is calculated. The charging duration is obtained by the formula (2)-(5).

Step 4) According to different charging powers, the charging process of the electric vehicle is divided into $\Delta t$ stages. The charging time of each stage of the battery is calculated according to the $\mathrm{SOC}_{\mathrm{i}}$ of each stage.

Step 5) Each stage of the EV power and harmonic coupling admittance matrix model parameters are called. The daily charging load power and each current of a single EV are obtained by bringing in the charging time results of steps 1 to 4 .

Step 6): The basic information in step 1) is assigned by the statistical data. Steps 1) to 5) are repeated, then the charging power and harmonic current corresponding to the plurality of electric vehicles $24 \mathrm{~h}$ are superimposed.

\section{Case Analysis}

\subsection{Single electric vehicle charging prediction result}

Using the proposed model, the resistance, power and secondary current variations of the full charging process are predicted as shown in Figure 2. Among them, the EV battery capacity is 
selected as $30 \mathrm{kWh}$, the maximum driving range is $160 \mathrm{~km}$, and the rated charging power is $9 \mathrm{~kW}$. In this paper, 10 minutes is taken as a stage, and the whole process of charging is 270 minutes. The first 150 minutes is a constant current charging process, and the resistance is basically unchanged. The maximum charge power reaches $9 \mathrm{~kW}$ at $150 \mathrm{~min}$, and the THD is $17.21 \%$. The remaining 120 minutes are the constant voltage stage. The resistance is gradually increased, the power and current are decreased, and the harmonic content is increased. The prediction results of this model are basically consistent with the measured results, and the results are more accurate than the constant power model.
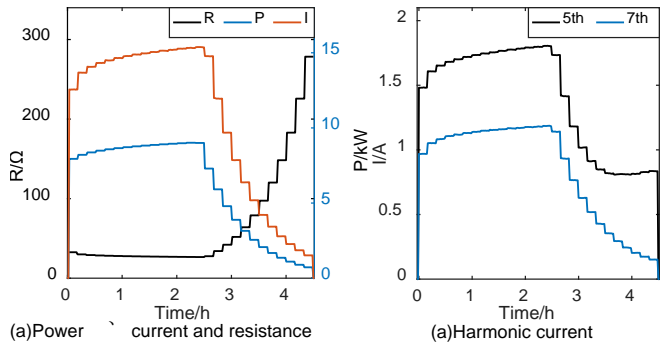

Figure 2. The curve of resistance, power and current of a single EV during the whole charging process.

\subsection{Scaled electric vehicle charging prediction result}

Residential area charging time is normally distributed $N(19,1.52)$, and the daily mileage distribution is $N(3.7,0.9)$ in residential area of private car working day, according to the statistical results [5]. The daily charging power and harmonic current curve of 50 electric vehicles with different battery performance, charging mode and power are shown in Figure 3. The EV charging time is from 15:40 to 6:00 the next day in this area. The power and THD is high, and it is consistent with the peak time of residential electricity consumption.

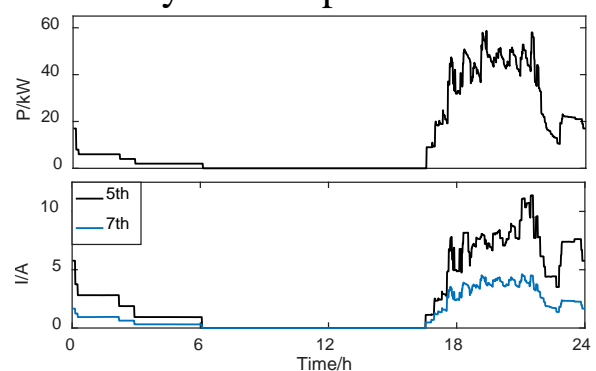

Figure 3. Daily variation of charging power and harmonic current of $50 \mathrm{EVs}$.

\subsection{Residential area load assessment results before and after EVs access}

The power and the current parameters of the electric vehicles before and after the access are shown in Table 1 and Table 2 for the 50 households with an electric vehicle occupancy rate of $80 \%$. The thresholds of power, fundamental current and 5th and 7th harmonic currents are selected as $35 \mathrm{~kW}, 50 \mathrm{~A}, 5 \mathrm{~A}$ and $1.5 \mathrm{~A}$, respectively. It can be seen that after the electric vehicle is connected, the load peak-to-valley difference is obviously improved. The length of time each parameter exceeds the threshold is multiplied. The harmonic content increased from $12.11 \%$ to $24.64 \%$. Therefore, it has a greater impact on the power grid. 
Table 1. Comparison of residential load power parameters under different working conditions.

\begin{tabular}{cccccc}
\hline & $\begin{array}{c}\text { Peak } \\
\text { /kW }\end{array}$ & $\begin{array}{c}\text { Peak and } \\
\text { valley } \\
\text { difference/kW }\end{array}$ & Mean/kW & $\begin{array}{c}\text { Standard } \\
\text { deviation } \\
/ \mathbf{k W}\end{array}$ & $\begin{array}{c}\text { Time } \\
\text { beyond } \\
\text { threshold } \\
/ \mathbf{m i n}\end{array}$ \\
\hline $\begin{array}{c}\text { Residential area } \\
\text { Residential area within EV }\end{array}$ & 36.68 & 34.08 & 8.41 & 7.11 & 6 \\
\hline
\end{tabular}

Table 2. Comparison of residential load current under different working conditions.

\begin{tabular}{cccccccccc}
\hline & \multicolumn{3}{c}{ Peak/A } & \multicolumn{3}{c}{ Mean/A } & \multicolumn{3}{c}{$\begin{array}{c}\text { Time beyond } \\
\text { threshold/min }\end{array}$} \\
\cline { 2 - 10 } & 1st & 5th & 7th & 1st & 5th & 7th & 1st & 5th & 7th \\
\hline $\begin{array}{c}\text { Residential area } \\
\text { Residential area within } \\
\text { EV }\end{array}$ & 61.18 & 5.09 & 1.71 & 10.85 & 1.68 & 0.29 & 8 & 1 & 18 \\
& 145.99 & 15.23 & 6.18 & 27.96 & 5.62 & 2.84 & 307 & 316 & 399 \\
\hline
\end{tabular}

\section{Conclusion}

The charging time of EVs has been simulated based on Monte Carlo method, and the evaluation model of EV power and harmonics has been established in this paper. The EVs are classified according to different battery performances and different charging methods, so the diversity of user charging behavior can be considered. The charging stage of the EV is divided according to the actual charging power. The power and current model of harmonic admittance matrix varying with SOC are established. Therefore, the accuracy of the charging power and current prediction model has been improved. The charging power and harmonics of single and large-scale electric vehicles are predicted by using the method proposed in this paper, and the electricity consumption in residential areas with or without EVs is compared. The impact of EV charging on the grid can be quantified, and the basis of grid operation scheduling and harmonic control can be provided.

\section{Acknowledgments}

This work was financially supported by National Natural Science Foundation of China (51577108) and Future Plan for the Young Scholars of Shandong University (2016WLJH07).

\section{References}

[1] A. Ashtari, E. Bibeau, S. Shahidinejad and T. Molinski, PEV Charging Profile Prediction and Analysis Based on Vehicle Usage Data, J. Sci. Commun. 3 (2012) 341-350.

[2] K. Qian, C. Zhou, M. Allan and Y. Yuan, Modeling of Load Demand Due to EV Battery Charging in Distribution Systems, J. Sci. Commun. 26 (2011) 802-810.

[3] D. Tang, P. Wang. Probabilistic Modeling of Nodal Charging Demand Based on Spatial-Temporal Dynamics of Moving Electric Vehicles, J. Sci. Commun.7 (2016) 627-636.

[4] S. Bae, A. Kwasinski. Spatial and Temporal Model of Electric Vehicle Charging Demand, J. Sci. Commun. 3 (2012) 394-403.

[5] A. Santos, N. Mcguckin, H.Y. Nakamoto and D. Gray, Summary of Travel Trends: 2009 National Household Travel Survey, J. Demographics. (2010).

[6] K. Clement-Nyns, E. Haesen, J. Driesen, The Impact of Charging Plug-In Hybrid Electric Vehicles on a Residential Distribution Grid, J. Sci. Commun. 25 (2010) 371-380. 